\title{
Towards a Better Understanding of Silicon Heterojunction Solar Cells
}

\author{
Stefaan De Wolf, Jérôme Damon-Lacoste, Luc Fesquet, Gregory Choong, and Christophe Ballif
}

Ecole Polytechnique Fédérale de Lausanne (EPFL), Breguet 2, CH-2000 Neuchâtel, Switzerland

\section{Introduction}

Semiconducting heterostructures increasingly attract attention for electronic junction formation in crystalline silicon ( $c$-Si) wafer-based solar cells. A key point of such a device is the displacement of highly recombination-active (ohmic) contacts from the crystalline surface by insertion of a film with wide bandgap. To reach the full device potential, the heterointerface state density should be minimal. Practically, hydrogenated amorphous silicon $(a-\mathrm{Si}: \mathrm{H})$ films of only a few nanometer thin are appealing candidates for this: Their bandgap is wider than that of $c$-Si and, when intrinsic, such films can reduce the $c$-Si surface state density by hydrogenation. In addition, these films can be doped relatively easily, either $n$ - or $p$-type, allowing for the fabrication of electronically abrupt $p-n$ and low-high heterojunctions $(\mathrm{HJ})$.

For such films, however, to simultaneously fulfill both the surface-passivation and the doping requirements has been proven to be challenging. Hence, typically, a fewnanometer thin intrinsic buffer layer is inserted between the $c$-Si surface and the doped $a$-Si:H films for device fabrication. For HJ solar cells featuring such stacked film structures, impressive large-area $\left(>100 \mathrm{~cm}^{2}\right)$ energyconversion efficiencies $(\sim 23 \%)$ have been reported by Sanyo, Japan [1]. Despite this result, the fundamental origin of the poor passivation of the doped $a-\mathrm{Si}: \mathrm{H} / c-\mathrm{Si}$ interface is not yet fully understood.

In this article, we focus on the role that such buffer layer plays for the interface passivation. For this, we show that post-deposition annealing offers in a straightforward way a single parameter to vary both electronic and material properties of the samples under study.

\section{Intrinsic a-Si:H film passivation}

Firstly, we argue that for an atomically sharp intrinsic $a$-Si:H / $c$-Si interface, the electronic interface passivation quality usually greatly benefits from post-deposition annealing at low temperatures $\left(<250{ }^{\circ} \mathrm{C}\right)$ [2]. Figure 1 shows how the value for the effective carrier lifetime, $\tau_{\text {eff }}$ changes over time when bifacially $a$-Si:H passivated $c$-Si wafers are subjected to isothermal annealing. It can be seen that irrespective of the film deposition temperature, $T_{\text {depo, }}$, the shown data can be fitted with satisfying accuracy to stretched exponentials of the form

$$
\tau_{e f f}\left(t_{a n n}\right)=\tau_{e f f}^{S S}\left[1-\exp \left[-\left(\frac{t_{a n n}}{\tau}\right)^{\beta}\right]\right]
$$

where $\beta$ is the dispersion parameter $(0<\beta<1)$, and $\tau$ effective time constant, respectively. For bulk $a$-Si:H material, defect reduction following stretched exponentials was explained in the past as arising either from dispersive (i.e. time dependent) hydrogen diffusion [3], or from retrapping included hydrogen motion [4]. For the $a-\mathrm{Si}: \mathrm{H}(i)$ / $c$-Si interface, based on the latter interpretation, it has been argued that the annealing induced passivation may originate from a transfer of hydrogen from a higher hydride state in the $a-\mathrm{Si}: \mathrm{H}$ film (close to the interface) to a monohydride $c$ $\mathrm{Si}$ surface state [5]. Consequently, the $a-\mathrm{Si}: \mathrm{H}(i) / c-\mathrm{Si}$ interface passivation is likely due to chemical surface state passivation, rather than a field effect.

\section{Doped $a-\mathrm{Si}: \mathrm{H}$ film passivation}

Secondly, we argue that in case the buffer layer is covered by a doped $a$-Si:H overlayer, annealing may actually be detrimental to the interface passivation. This especially the case for $p$-type $a$-Si:H films [6]. Indeed, although doped films may yield a field effect at the interface, their electronic passivation properties are often found to be inferior, compared to those of their intrinsic counterparts. This is shown in figure 2: Panel (a) shows the change in surface passivation quality, expressed by $\tau_{\text {eff, }}$ as a function of an isochronal stepwise annealing treatment for respectively a few nm thin intrinsic, $n^{+}$-, and $p^{+}$-doped single film $a$-Si:H / $c$-Si hetero-structures. Whereas annealing up to

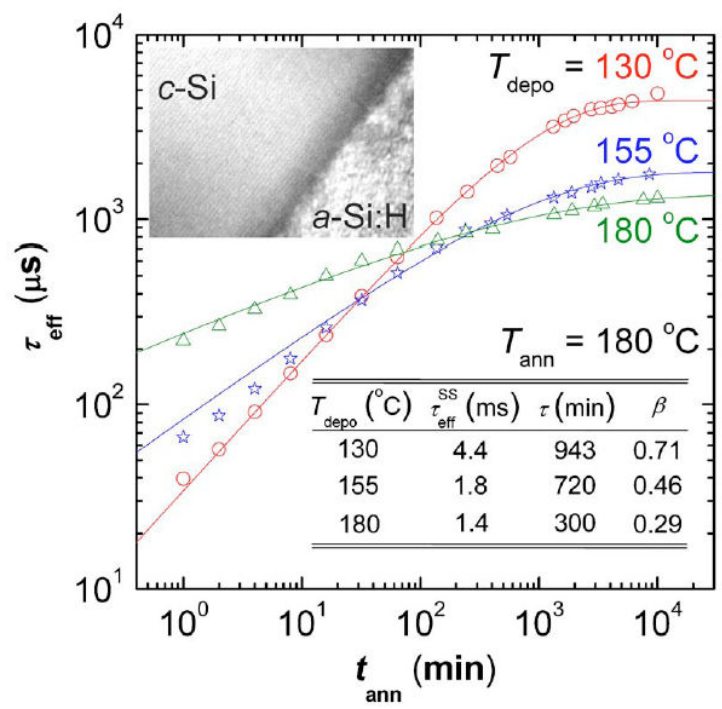

FIG. 1. Measured values for $\tau_{\text {eff }}$ as function of $t_{\text {ann }}$ for a $\sim 3.0 \Omega$.cm floatzone wafer bifacially passivated by $\sim 50 \mathrm{~nm} a-\mathrm{SiH}(i)$ films. The cases for three different deposition temperatures are shown. Evaluation was performed at $\Delta n=\Delta p=1.0 \times 10^{15} \mathrm{~cm}^{-3}$. The annealing temperature was fixed at $180{ }^{\circ} \mathrm{C}$. Symbols represent measured data. Solid lines represent stretchedexponential fits to the data. Values for the fitting parameters are given in the inset table. The inset HR-TEM micrograph is taken from one of the interfaces of a mirror-polished $c$-Si (100) sample featuring $a$-Si:H films for which $T_{\text {depo }}=200{ }^{\circ} \mathrm{C}$. Data taken from [5]. 


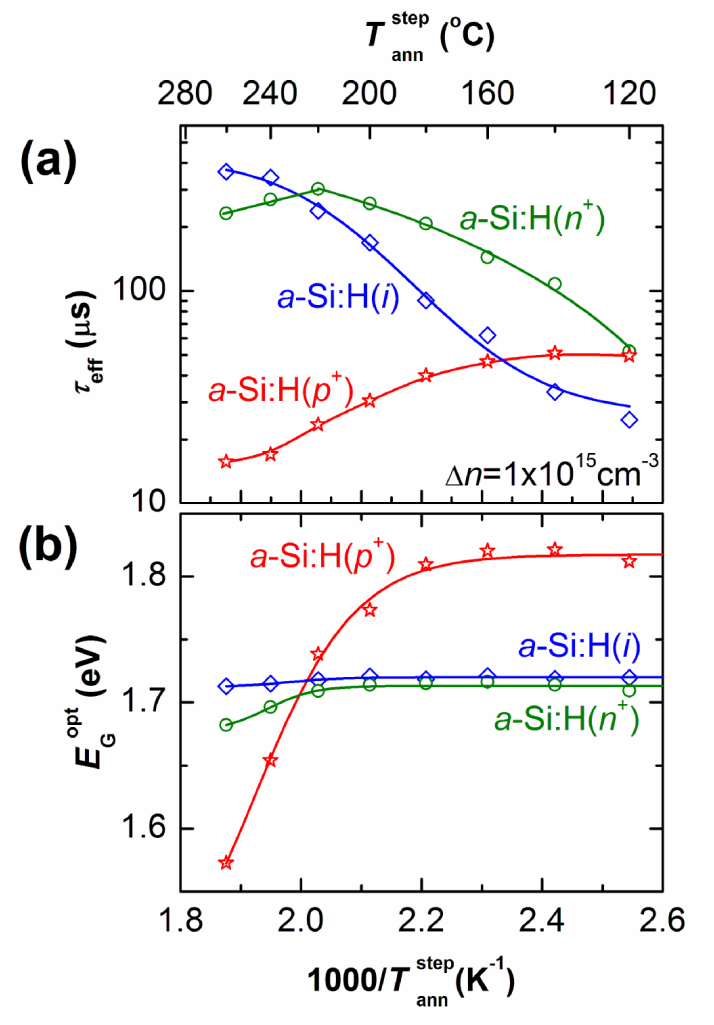

FIG. 2. (a) Influence of stepwise annealing on a few $\mathrm{nm}$ thin single film $a$-Si:H / $c$-Si interface passivation quality, expressed by $\tau_{\text {eff }}$ (at $\Delta n=\Delta p=$ $\left.1.0 \times 10^{15} \mathrm{~cm}^{-3}\right)$. No intrinsic buffer layers are present underneath the doped films. (b) Influence of stepwise annealing treatment on the optical bandgap $E_{\mathrm{G}}{ }^{\text {opt }}$ for similar films as in (a), extracted from spectroscopic ellipsometry measurements. Symbols represent experimental data, the lines are guides for the eye. Data taken from [9].

$260^{\circ} \mathrm{C}$ has a beneficial effect on the surface passivation properties of $a-\mathrm{Si}: \mathrm{H}(i)$ films, the same treatment is seen to result immediately in losses for the $a-\mathrm{Si}: \mathrm{H}\left(p^{+}\right)$case.

For $a$-Si:H $\left(n^{+}\right)$films, initially an improvement in passivation quality can be seen. Nevertheless, annealing above $220^{\circ} \mathrm{C}$ results also here in losses. Panel (b) of the same figure shows how the optical bandgap, $E_{G}^{o p t}$, extracted from spectroscopic ellipsometry measurements, of these films changes during the annealing treatment. Interestingly, for all three cases, the onset of passivation degradation (if present) seems to coincide with the onset of a collapse of $E_{G}^{\text {opt }}$. This may point at defect generation in the amorphous host matrix.

The latter phenomenon is attributed to the position of $E_{\mathrm{F}}$ within the bandgap, influencing the generation of (native) compensation defects in the semiconductor, counteracting intentional doping. Fundamentally, as the bandgap of a semiconductor increases, often it becomes increasingly difficult to dope it in a symmetric way (both $n$ - and $p$-type). This may originate from the generation of compensation defects, depending on $E_{\mathrm{F}}$, according to the relation:

$$
\Delta H^{(D, q)}\left(\mu, E_{F}\right)=q E_{F}+n_{D}\left(\mu_{D}-\mu_{H}\right)+\Delta E_{b},
$$

describing the formation enthalpy of dopant $D$ of charge state $q$ in host semiconductor $H$ [7]. Here, $\mu_{\mathrm{D}}$ and $\mu_{\mathrm{H}}$ are the chemical potentials of the dopants and host, $\Delta E_{\mathrm{b}}=$ $E$ (host+defect) - E(host), and $E$ is the total energy. Deliberate $p$-type (n-type) doping of the material by acceptors (donors) will shift $E_{\mathrm{F}}$ towards the valence band maximum (conduction band minimum) and decrease the formation energy of native donors (acceptors) to a point where they are created spontanously. Usually, $E_{\mathrm{F}}$ can not be pushed beyond this point (it will be pinned) due to the occuring electronic compensation. Consequently, in $a$-Si:H, the described $E_{\mathrm{F}}$ dependent defect generation may actually originate from its bandgap width as well. Here, the defect most likely formed by either type of doping is the amphoteric Si dangling-bond (DB), through $\mathrm{Si}-\mathrm{H}$ rupture. At equilibrium, this defect may be neutral $\left(D_{3}{ }^{0}\right)$, positively $\left(D_{3}{ }^{+}\right)$or negatively $\left(D_{3}^{-}\right)$charged, according to the position of $E_{\mathrm{F}}$, accomodating respectively 1,0 and 2 electrons.

\section{Stacked film passivation: role intrinsic buffer layer}

The presence of such a doped $a$-Si:H overlayer on an intrinsic $a$-Si:H buffer layer may also shift the Fermi-energy in the latter film. Hence, the energy required for $\mathrm{Si}-\mathrm{H}$ bond rupture likely is lowered slightly in the underlying intrinsic buffer layer as well. Principally, this may be the reason why the presence of a doped overlayer can be detrimental to the $a$-Si:H(i) / c-Si interface passivation during annealing, albeit to a lesser extent than for structures lacking such buffer layers $[8,9]$.

\section{Conclusions}

In this article, we have shown that low-temperature annealing studies can be a useful tool in the further understanding of silicon heterojunction solar cells. The intrinsic $a$-Si:H buffer layer provides a mono-hydride $c$-Si surface termination. Doped layers have been demonstrated often to passivate poorly due the presence of doping compensating defects.

\section{Acknowledgements}

This work was supported by the European Community's Seventh Framework Programme (FP7/2007-2013) under the Hetsi Project (Grant Agreement No. 211821), and Axpo Holding AG, Switzerland in the frame of the Axpo Naturstrom Fond.

\section{References}

[1] Sanyo, Japan, press release (2009).

[2] S. De Wolf and M. Kondo, Appl. Phys. Lett. 90, 042111 (2007).

[3] J. Kakalios, R.A. Street, and W.B. Jackson, Phys. Rev. Lett. 59, 1037 (1987).

[4] C.G. Van de Walle, Phys. Rev. B 53, 11292 (1996).

[5] S. De Wolf, S. Olibet, and C. Ballif, Appl. Phys. Lett. 93, 32101 (2008).

[6] S. De Wolf and G. Beaucarne, Appl. Phys. Lett. 88, 022104 (2006).

[7] A. Zunger, Appl. Phys. Lett. 83, 57 (2003).

[8] S. De Wolf and M. Kondo, Appl. Phys. Lett. 91, 112109 (2007).

[9] S. De Wolf and M. Kondo, J. Appl. Phys. 105, 103707 (2009). 